\title{
Several Issues on the Development of Mongolian Whole Media Fusion News Production Practice Teaching Platform
}

\author{
Huiru Deng ${ }^{1}$, Liping Zhu ${ }^{2, a, *}$ \\ ${ }^{1}$ Information Engineering College, Minzu University of China, Beijing 100081, China \\ ${ }^{2}$ National Language Resource Monitoring and Research Center on Minority Language, Beijing \\ 100081, China \\ azlp6681@sina.com
}

Keywords: Media Convergence, Mongolia Text, Video Download, Bootstrap Framework, Font Awesome.

\begin{abstract}
In order to cater to the Internet education and the current industry news media trend, developed minority language news gathering and editing website. Mongolian whole media fusion news production practice teaching platform is a teaching site aimed at Mongolian students, on which students can search and browse instant news, download video, add video editing captions and post back to the server. In this paper, some difficulties encountering in the development of the Mongolian whole media fusion news production practice teaching platform are discussed, including vertical display of the Mongolian text, download, and preview of video and realization of better users' experience. Convenient and innovative solutions are proposed and functioned for these problems, achieving good effects in practice.
\end{abstract}

\section{Introduction}

In the era of Internet, the media industry comes into the era of media integration, traditional journalism changes to a certain extent [1]. A variety of media manifest a developing trends of the integration of multi-function [2], so talent training for whole media news becomes the focus of the department of journalism in most universities [3]. Many works concentrate on the research and development of the teaching and training platform with whole media integration $[4,5,6]$. However, there are few teaching and training platforms for the minority whole media personnel, and website developed by Mongolian language for is very few. In this paper, the problems encountered in the development of the Mongolian whole-media media integration news production teaching and training platform are summarized.

\section{Vertical display of the Mongolian text}

Web page text in the Chinese and English web site are generally displayed in accordance with the order from left to right, but Mongolian text is displayed in accordance with the order from top to bottom. In [7], the Mongolian web page text to be vertical displayed are first output from right to left, and then transposed to display. In [8], the text is first displayed in a single column, and then the text next to it is inserted at the right side. Repeating the process can realize the vertical display of the Mongolian text from left to right. In this article, the properties in CSS3 are employed to display text vertically. Writing-mode [9], as a private property of the early IE browser, has multiple values, among which lr-tb, tb-rl and tb-lr are often used. (1) lr-tb represents the display mode of left to right and top-down. The page contents display in the horizontal direction from left to right and then from top to bottom. (2) tb-rl represents the display mode of top-down and right to left. The contents on the page are displayed vertically from top to bottom and then from right to left. (3) tb-lr represents the display mode of top to bottom and left to right. The contents on the page are displayed vertically from top to bottom and then from left to right. These properties are accepted by $\mathrm{W} 3 \mathrm{C}$ as standard properties. They are modified as one attribute with three values, i.e., writing-mode attribute with values of horizontal-tb, vertical-rl and 
vertical-lr, respectively, which are similar to $1 r-r b$, tb-rl and tb-lr, respectively. According to the special output format of Mongolian text, the vertical-lr is adopted. As is shown in Table 1 below, among the mainstream browsers, except that IE browser still supports the private attribute value of tb-lr, other browsers all support vertical-lr.

Table 1.Browsers supporting the writing-mode attribute

\begin{tabular}{|c|c|c|c|c|c|}
\hline Browser & IE & Chrome & Firefox & Opera & Safari \\
\hline writing-mode & tb-lr & vertical-lr & vertical-lr & vertical-lr & vertical-lr \\
\hline
\end{tabular}

\section{Video download}

The news production practice platform requires download the video from the web, so that students can edit the news, such as adding subtitles and so on. How to achieve video download from the web is seldom discussed in the existing literature. Two file download methods are mentioned in [10]. One uses a hyperlink directing at the file storage location, but this method has a problem. The browser will automatically open the file when clicking on the hyperlink. The other method uses the java file input and output stream to download files, but embedding the java code into the jsp pages is likely to cause confusion. The code readability may be reduced which is not conducive to post-maintenance. In this paper, servlet is adopted to download video files. As servlet is implemented at the server-side and separated from the html, it is more efficient and easily portable.

Click on the download button to submit form, the web page will to send the location of the file to be download at the server-side to the DownloadServlet through the form. The implementation code is as follows.

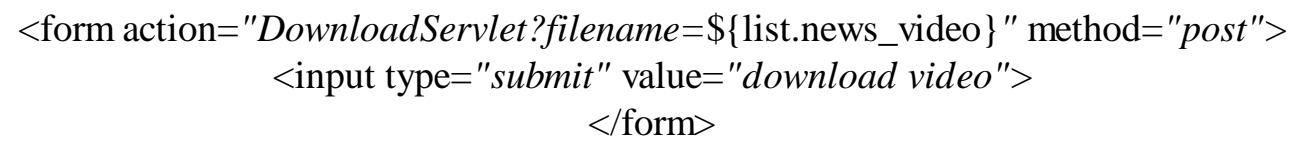

Servlet receives a request from the web page, and then return file in the form of data stream to the client. Implementation code is as follows:

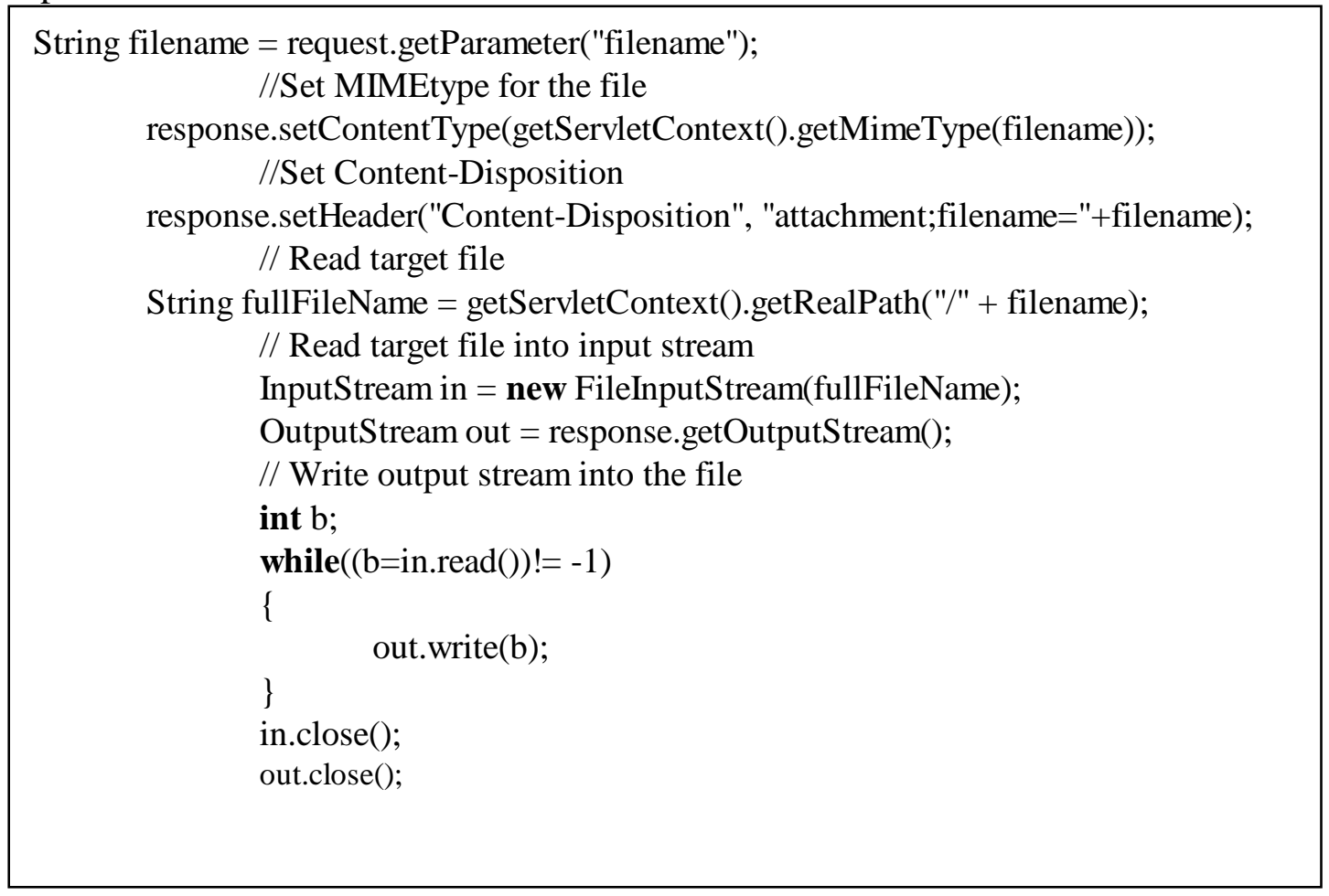




\section{Application of the Bootstrap fileinput}

The whole media platform developed in this paper is mainly used for teaching. Students can choose and download certain video from a video library, edit subtitles on the video, and then upload the video, so that teachers can check the students' task. In the upload process, it needs to add a preview function on the page in order to judge whether the selected video is the target video. Common file upload method sets the type of the <input> tag as file. But this method can only select the file, but can't preview the file. In our schemes, the Bootstrap fileinput plugin is adopted for file upload.

Bootstrap [11] is an open source front-end framework based on HTML5, CSS3 [12], and JavaScript, which includes a large number of web components. Bootstrap fileinput is a file upload component achieving file upload and multi-file upload preview. Following is the approach of its usage. First download the file fileinput.css and fileinput.js. If it is to show the Chinese text, then it also needs to download fileinput_locale_zh.js for chinesization. Then relevant documents are introduced in the $<$ head>. The main code is as follows:

Then write the <input> tag in the 〈body> tag, and add the appropriate attributes. The implementation code is as follows:

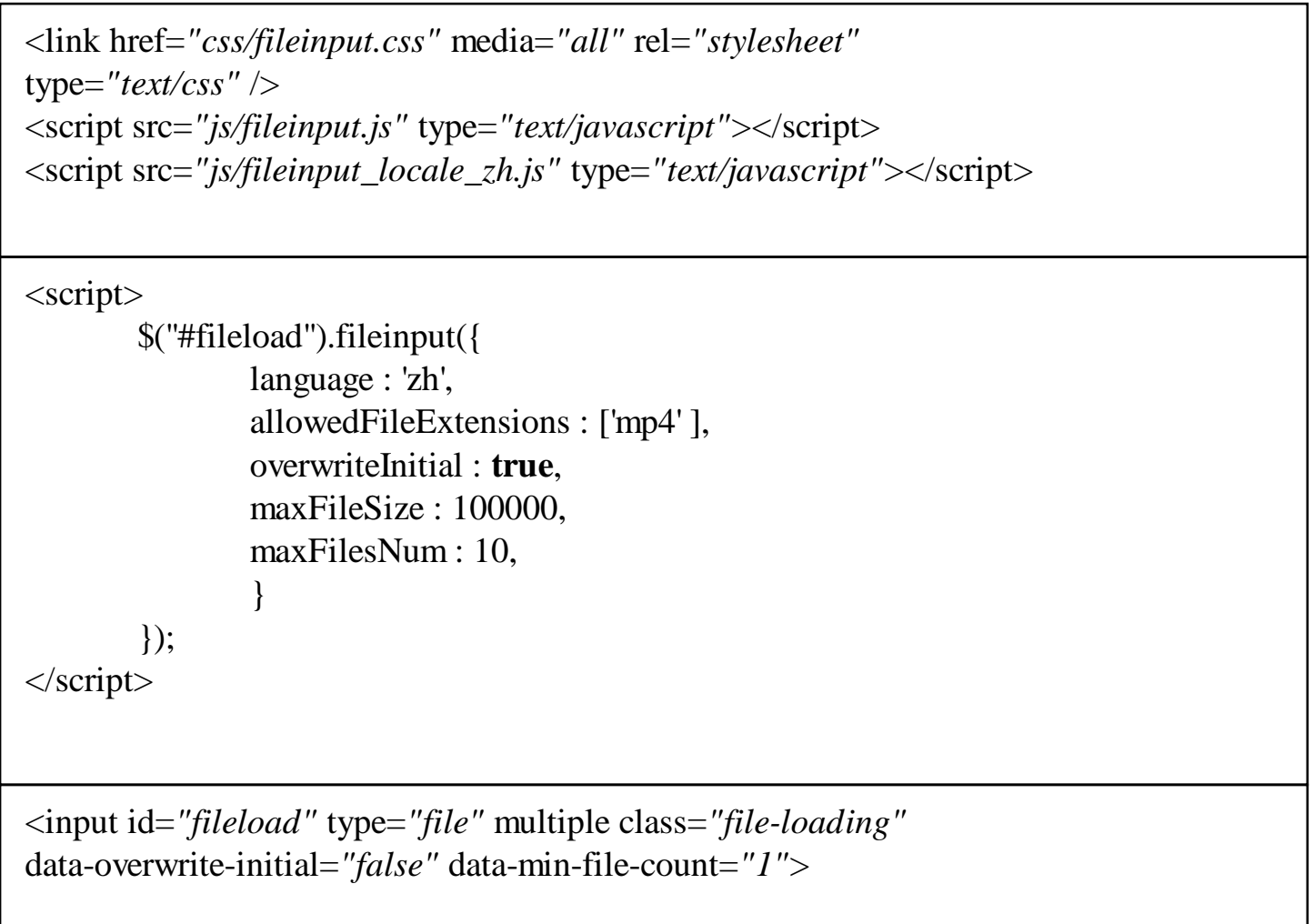

\section{Use of Fone Awesome font graphics library}

The icon is a graphic that has an identifying nature and indicative meaning. It is highly concentrated, fast in conveying information, and easy to remember [13]. In the web design, the icon is a crucial component. The rational use of icons makes the site flexible and simple and enhances the user experience. In the platform development, Fone Awesome font graphics library is used to make students better understand the function of platform.

Fone Awesome is designed by Dave Grandy which is widely used in web front-end development [14]. The latest version is Version 4.7.0. The characteristics of the Fone Awesome are as follows:

1) It is a font with 675 icons.

2) Not depend on JavaScript.

3) Unlimited zoom of the icon size.

4) Completely free.

5) Color, size, shadow or any other effects can be easily show as long as CSS support. 
6) Fone Awesome was originally designed for Bootstrap framework, but also compatible with other frameworks.

Following is the procedures for the use of the Fone Awesome.

First, download Fone awesome font graphics package font-awesome.min.css, and then introduce font-awesome.min.css into the html <head > tag, i.e., <Link rel $=$ "stylesheet" href = "css / font-awesome.min.css" $\rangle$. Next, use $<\mathrm{i}\rangle$ in the $\langle$ Body $\rangle$ tag, name its class name prefix fa, and add the name of the icon. Then the icon can be displayed in the page, i.e., $<$ I class $=$ "fa fa-location-arrow" $></$ i>.

\section{Concluding remarks}

The Mongolian news teaching platform is developed on the basis of media fusion. The solutions to some problems encountered in the development process are puts forward. It achieves the vertical display of Mongolian text on the website by CSS style, the video download through the servlet, the video upload and preview via Bootstrap fileinput component and adopts icon in graphics tool library of Fone awesome as the graphical mark of the site. The solutions are not confined to the development of teaching sites, they can also be used in the development of other various Mongolian-wide media sites.

\section{Acknowledgements}

The project is funded by the "Natural Science Foundation Workshop of MUC" and Key project of Natural Science Foundation under grant no. 61331013.

\section{References}

[1]. Guang Li. Linyi University. The strategy analysis of traditional newspaper industry transformation during the all media context. Editorial Friend, 2015(10):22-25.

[2]. Jia-li Ying. Lanzhou University. Research on news report of People's Daily and its new media platform in media convergence environment. 2015.

[3]. Jing Yan. On the cultivation of all - media journalism talents in the age of media. Press, 2011 (05): 150-153.

[4]. Pin-jing Su. Development strategy of news websites in the background of media. China Publishing, 2016 (04): 36-38.

[5]. Yu-qing Liu, Ying Du. Discussion on the construction of all - media experimental platform in news communication teaching. Experimental technology and management, 2012, 29(11):169-173.

[6]. Rui Song, Lulu Zou. Research on production and operation platform of all - media convergence. TV Technology, 2015, 39 (24): 69-71.

[7]. Ya Tu, Xin-ming Ye. Study on Some Problems in Dynamic Mongolian Website Construction. Journal of Inner Mongolia University: Natural Science Edition, 2007, 38 (5): 591-594.

[8]. Haizhu Li. A study of font embedding and vertical text in Mongolian. Journal of Inner Mongolia Normal University :Natural Science Edition, 2005, 34 (01): 48-49.

[9]. Information on: http: //css.doyoe.com/.

[10]. Sun Qing Gele. JSP in the realization of file downloads and statistical downloads. File, 2012 (6): 78-79.

[11]. Information on: http://www.bootcss.com/.

[12]. Frain B . Responsive web design with HTML5 and CSS3 : build responsive and future-proof websites to meet the demands of modern web users. 2015.

[13]. Xinxin Jing. Analysis of icon design in the application of interactive interface design. Art and Life - Wen Hai Yi Yuan, 2012 (11): 218-218.

[14]. Information on: http: //fontawesome.dashgame.com//. 